\title{
Theoretical and experimental analysis of the core sampling method : Reducing diffusional losses in aerosol sampling line
}

\section{Fu, Yueyun}

2019-07-03

Fu , Y , Xue , M , Cai , R , Kangasluoma , J \& Jiang , J 2019 , ' Theoretical and experimental analysis of the core sampling method : Reducing diffusional losses in aerosol sampling line ' , Aerosol Science and Technology , vol. 53 , no. 7 , pp. 793-801 . https://doi.org/10.1080/02786826.2019.1608354

http://hdl.handle.net/10138/322358

https://doi.org/10.1080/02786826.2019.1608354

acceptedVersion

Downloaded from Helda, University of Helsinki institutional repository.

This is an electronic reprint of the original article.

This reprint may differ from the original in pagination and typographic detail.

Please cite the original version. 


\title{
Theoretical and experimental analysis of the core sampling method: reducing diffusional losses in aerosol sampling line
}

\author{
Yueyun $\mathrm{Fu}^{1 \dagger}$, Mo Xue ${ }^{1 \dagger}$, Runlong $\mathrm{Cai}^{1}$, Juha Kangasluoma ${ }^{2,3}$, and Jingkun Jiang ${ }^{1 *}$ \\ ${ }^{1}$ State Key Joint Laboratory of Environment Simulation and Pollution Control, \\ School of Environment, Tsinghua University, 100084 Beijing, China \\ ${ }^{2}$ Aerosol and Haze Laboratory, Advanced Innovation Center for Soft Matter Science \\ and Engineering, Beijing University of Chemical Technology, Beijing, China \\ ${ }^{3}$ Institute for Atmospheric and Earth System Research / Physics Faculty of Science, \\ University of Helsinki, P.O. Box 64, 00014 Helsinki, Finland
}

†: Yueyun Fu and Mo Xue contributed equally to this study.

*Correspondence to: J. Jiang (jiangjk@tsinghua.edu.cn)

To be submitted to

Aerosol Science and Technology

March, 2019 


\begin{abstract}
Core sampling method (extracting a portion of a flow from the core of the flow) will reduce diffusional losses of highly diffusive species (e.g., aerosol nanoparticles, ions, and gases) when transporting them through a sampling tube. Revealing parameters governing the sampling efficiency of a core sampling system, $\eta_{\text {sam }}$, helps to design the apparatus and to optimize its performance. In this study, we report an analytical solution for quantifying the $\eta_{\text {sam }}$ by solving the convection diffusion equation of laminar flow field. The analytical results were experimentally evaluated using 1-5 nm tungsten oxide nanoparticles. $\eta_{\text {sam }}$ is governed by a dimensionless loss parameter and the transport-tosample flow ratio. Theoretically predicted values for $\eta_{\text {sam }}$ agree with experimental results, e.g., the relative deviation is within $5 \%$ when the value for the loss parameter is less than 0.1. The core sampling method is recommended to work at the loss parameter less than 0.1 such that $\eta_{\text {sam }}$ is equal or close to the maximum value of unity and is also insensitive to variations in sampling conditions. How to apply the findings in designing and optimizing a core sampling system was discussed. A core sampling apparatus was then designed and experimentally evaluated. Its sampling efficiency was shown to be significantly higher than those of a tee, a cross fitting, and a Y fitting when the same sampling conditions were used.
\end{abstract}




\section{Introduction}

When sampling highly diffusive species such as aerosol nanoparticles, ions, molecular clusters, and gases, reducing their diffusional losses is a key for improving the sampling efficiency. Increasing the total flow rate by using a transport flow is a commonly used approach to improve the sampling efficiency. When adding the transport flow to a sampling line, a connector such as a tee, a Y fitting, or a cross fitting is often used. The total flow is separated by the connector into the transport flow and the sample flow. In such cases, the connector only functions as a flow splitter. However, a properly designed connector can further improve the sampling efficiency apart from just adding the transport flow. In a cylindrical sampling tube, highly diffusive species are typically more abundant in the core region around the flow centerline, as their concentrations typically decrease along the radial direction and reach zero on the tube wall. Accordingly, sampling from the central region of a sampling line, i.e., the core sampling method, is assumed to be a more effective approach to improve the sampling efficiency in comparison to a conventional connector.

The core sampling method was used in the capillary inlet of an ultrafine condensation particle counter (Stolzenburg and McMurry 1991), the sampling inlet of an electrical mobility spectrometer for sub-3 nm atmospheric particles (Jiang et al. 2011a), and the vacuum entrance aperture of a chemical ionization mass spectrometer (Eisele and Tanner 1993). Recently, Kangasluoma et al. (2016) designed a core sampling apparatus for nanoparticles and measured its sampling efficiency at a fixed sample flow rate. However, the sampling efficiency of this apparatus varies with sampling conditions including the diffusivity of the sampled species, the length of the tube, and the transport flow rate. Revealing parameters governing the sampling efficiency of a core sampling system through theoretical and experimental analysis is helpful for designing the apparatus and for optimizing its performance. In addition, the sampling efficiency is needed to derive the concentration of sampled species at the entrance of the sampling system.

For a straight cylindrical tube, the convection diffusion equation (CDE) quantitatively describes the spatial distribution of the concentration inside it. Laminar flow within the tube is a commonly used assumption since most applications are designed to avoid turbulence (i.e., Reynolds number is less than 2000) (Kundu et al. 2012). For typical sampling lines, the influence of the entrance length of a flow that completely transforms from the plug flow to the Hagen-Poiseuille flow is not significant compared to the predicted values under laminar flow assumption (Alonso et al. 1997). Under the laminar flow condition, the CDE is usually simplified as a Graetz problem, which is analogous 
to the analysis of heat and mass transfer (e.g., Eckert and Drake 1987; Sellars et al. 1954). When several boundary conditions are met, the Graetz problem can be analytically solved to obtain the spatial distribution of concentration, and thus the average sampling efficiency at a cross sectional profile (e.g., Gormley and Kennedy 1948). Knopf et al. (2015) solved the Graetz problem with various boundary conditions to study the penetration of gaseous species and particles. With some adaptions, similar analysis can be applied to a core sampling system.

In this study, the sampling efficiency of the core sampling method is theoretically evaluated following the analysis of the Graetz problem. Its governing parameters are examined. The sampling efficiency of sub-5 $\mathrm{nm}$ tungsten oxide nanoparticles through a core sampling apparatus are measured at various conditions and compared with theoretical analysis. Applications in designing and optimizing the core sampling system are discussed and exercised with an example in comparison to conventional connectors (a tee, a cross fitting, and a Y fitting,).

\section{Theory}

Figure 1 shows a typical core sampling apparatus comprising two coaxial straight cylindrical tubes and its parameters. At the A-A profile (the cross section right at the entrance of the core sampling tube), sample flow is extracted into the core sampling tube from the core of the total flow, while the transport flow exits via the annulus between two tubes carrying a lower concentration of the diffusive species compared to the concentration in the sample flow.

The spatial distribution of these diffusive species upstream of the A-A profile is governed by the steady-state CDE. With several assumptions, CDE can be simplified and non-dimensionalized in a cylindrical coordinate as (see section 1 in the supplementary information (SI) for details):

$$
\begin{aligned}
& 2 \frac{\partial n(\tilde{r}, \tilde{z})}{\partial \tilde{z}}=\frac{1}{\left(1-\tilde{r}^{2}\right) \tilde{r}} \cdot \frac{\partial}{\partial \tilde{r}}\left(\tilde{r} \frac{\partial n(\tilde{r}, \tilde{z})}{\partial \tilde{r}}\right) \\
& n(\tilde{r}, 0)=1 \quad \text { for } \tilde{r}<1 \\
& n(1, \tilde{z})=0 \quad \text { for } \tilde{z} \geq 0 \\
& \partial n(\tilde{r}, z) /\left.\partial \tilde{r}\right|_{\tilde{r}=0}=0 \text { for } \tilde{z} \geq 0
\end{aligned}
$$

where $\tilde{n}=n / n_{0}, \tilde{r}=r / R$, and $\tilde{z}=\pi D z /\left(Q_{t}+Q_{s}\right) . n(r, z)$ is the concentration of diffusive species as a function of the spatial position in a cylindrical coordinate; $n_{0}$ is 
the concentration of the diffusive species at the entrance of the tubing which is often assumed to be uniform; $r$ is the radial position; $R$ is the inner radius of the outer tube; $z$ is the axial position; $D$ is the diffusivity of the sampled diffusive species; $Q_{t}$ is the transport flow rate; and $Q_{s}$ is the sample flow rate. The analytical solution of Eq. 1 is given as:

$$
\begin{aligned}
& n(\tilde{r}, \tilde{z})=\sum_{m=1}^{\infty} A_{m} e^{-\lambda_{m}{ }^{2}} e^{-\lambda_{m} \tilde{r}^{2} / 2} M\left(\frac{1}{2}-\frac{\lambda_{m}}{4}, 1, \lambda_{m} \tilde{r}^{2}\right) \\
& \mathrm{M}(a, b, X)=1+\sum_{i=1}^{\infty} \frac{a(a+1) \ldots(a+i-1)}{b(b+1) \ldots(b+i-1)} \frac{X^{i}}{i !}
\end{aligned}
$$

where $\mathrm{M}$ is the confluent hypergeometric function of the first kind (Subramanian 2012). $\lambda_{m}$ are zeros of $\mathrm{M}(1 / 2-\lambda / 4,1, \lambda)$ as solved by numerical methods (Mikhailov and Cotta 1997; Sellars et al. 1954). $A_{m}$ are multiplicative coefficients corresponding to each $\lambda_{m}$. The values of $A_{m}$ and $\lambda_{m}$ are predetermined constant values with respect to Assumptions 1-2 and Conditions 1-3 (section 2 in the SI).

A commonly used dimensionless loss parameter (e.g., Gormley and Kennedy 1948; Yook and Pui 2006), $\mu$, was adopted to characterize diffusional losses:

$$
\mu=\pi D L /\left(Q_{t}+Q_{s}\right)
$$

A similar loss parameter $\mu_{0}$ was defined for the sampling scenario without the transport flow:

$$
\mu_{0}=\pi D L / Q_{s}
$$

When the axial position $z$ is equal to the length $L$ (see Figure 1), $\tilde{z}$ is the same to $\mu$. Thus the concentration distribution at the A-A profile, $\tilde{n}(\tilde{r}, \tilde{z})$, can also be denoted as $\tilde{n}(\tilde{r}, \mu)$, i.e., the distribution at the A-A profile is only affected by the value of $\mu$.

Figure 2 illustrates the concentration profiles at different $\mu$ values. As the concentration distribution is solved with the initial condition of uniform concentration at the entrance of the apparatus. More curved concentration distribution is developed when $\mu$ is relatively large (e.g., $\mu=0.2$ ) and it has a plateau region when $\mu$ is relatively small (e.g., $\mu=0.01)$.

Re-establishment of the flow field happens upstream of the A-A profile due to flow splitting. For this disturbed region of non-parabolic flow field, Eq. 1 will give biased 
concentration distribution. However, the length for the flow developing upstream of the A-A profile is expected to be short and negligible compared to the tube length $L$, which is validated by simulation results (see the SI for further illustrations). Additionally, the flow disturbance exerted by the tube wall thickness of the core sampling tube is negligible due to the tapered inlet.

The core sampling efficiency, $\eta_{\text {sam }}$, is defined as the ratio of the average concentration of the core sample flow at the A-A profile to the average concentration of the total flow at the entrance of the whole sampling system. It can be obtained by integrating the product of $\tilde{n}(\tilde{r}, \tilde{z})$ and the parabolic air flow velocity distribution with respect to $\tilde{r}$ at the A-A profile. The dimensional and dimensionless expressions for $\eta_{\text {sam }}$ are given by Eqs. 5 and 6, respectively,

$$
\begin{gathered}
\eta_{\text {sam }}=f\left(D, L, Q_{t o t}, R\right)=\frac{\int_{0}^{\tilde{r}_{a} \cdot R} 4 \pi n\left(r / R, \frac{\pi D L}{Q_{t o t}}\right) \cdot \frac{Q_{t o t}}{\pi R^{2}} \cdot\left[1-(r / R)^{2}\right] r d r}{\int_{0}^{\tilde{r}_{a} \cdot R} 4 \pi n n \frac{Q_{t o t}}{\pi R^{2}}\left[1-(r / R)^{2}\right] r d r} \\
\eta_{\text {sam }}=f\left(\mu, \tilde{r}_{a}\right)=\frac{\int_{0}^{\tilde{r}_{a}} 4 \pi n(\tilde{r}, \mu) \cdot\left(1-\tilde{r}^{2}\right) \cdot \tilde{r} \cdot d \tilde{r}}{\int_{0}^{\tilde{r}_{a}} 4 \pi\left(1-\tilde{r}^{2}\right) \cdot \tilde{r} \cdot d \tilde{r}}
\end{gathered}
$$

where $f$ and $\tilde{f}$ are functions of dimensional and non-dimensionalized parameters, respectively; and the upper bound of integration $\tilde{r}_{a}$ is the non-dimensionalized radius where the sample flow and the transport flow split:

$$
\tilde{r}_{a}=\sqrt{1-\sqrt{1-Q_{s} /\left(Q_{t}+Q_{s}\right)}}
$$

$\tilde{r}_{a}$ decreases with an increasing transport-to-sample flow ratio $Q_{t}: Q_{s}$, and accordingly, $\eta_{\text {sam }}$ increases until it reaches the maximum of unity. The upper bound of integration is calculated based on that the highly diffusive species follow the splitting streamlines of a Hagen-Poisseuille flow within negligible length.

MATLAB (MathWorks Inc., Release 2018b) scripts are included in the SI for calculating the corresponding sampling efficiency for given $\mu$ and flow information. Note that $\tilde{n}(\tilde{r}, \mu)$ was solved based on the assumptions of fully developed laminar flow, negligible axial diffusion, and negligible length for flow splitting. For comparison, gridbased numerical results were obtained using a finite element analysis software package COMSOL Multiphysics (COMSOL Inc., Version 5.3) without the above assumptions. 
A core sampling apparatus consisting of a 1/2 inch outer tube and a 1/4 inch core sampling tube was simulated, where $Q_{s}$ is $0.8 \mathrm{LPM}$; $Q_{t}$ is $10 \mathrm{LPM}$; is $1.5 \mathrm{~m}$ or $0.5 \mathrm{~m}$; and $D$ is $0.1 \mathrm{~cm}^{-2} \mathrm{~s}^{-1}$. The analytically predicted $\eta_{\text {sam }}$ and numerical results agree within $\sim 5 \%$ deviation (see the SI for details).

\section{Experiment}

The sampling efficiency through a core sampling apparatus was experimentally characterized and compared to the analytical solution. Tungsten oxide particles were generated using a wire generator (Kangasluoma et al. 2015). It has a curled tungsten wire between two electrodes. The length and diameter of the tungsten wire are $12 \mathrm{~cm}$ and $0.4 \mathrm{~mm}$, respectively. The electrodes were powered by a $\sim 120 \mathrm{~W}$ DC power supply. Nitrogen (99.999\% purity) was used as the carrier gas and introduced into the wire generator at a flow rate of 20 LPM, which was controlled by a mass flow controller (MFC, Model D079E, Sevenstar Inc.). The generated tungsten oxide particles then entered a high resolution half-mini differential mobility analyzer (DMA, Fernández de la Mora and Kozlowski 2013). The sheath flow of the half-mini DMA was operated in a close loop for maintaining a stable operation. A cooling coil was installed on the sheath flow tubing to keep it at room temperature ( $293 \mathrm{~K})$. Classified monodisperse particles by the half-mini DMA then entered into a homemade four-way flow splitter. The two opposite outlets of the splitter were connected to two aerosol electrometers (TSI Inc., Model 3068B). This splitter was designed to avoid turbulence and to provide fair comparison for the two outlets. Details of the splitter are given in the SI. The pressure inside the four-way splitter was approximately the same to ambient pressure $(\sim 1 \mathrm{~atm})$. The tested core sampling apparatus was installed upstream of one aerosol electrometer. Then, the particle number concentration upstream and downstream of the core sampling apparatus, $N_{l}$ and $N_{2}$, were recorded by these two aerosol electrometers, respectively. The transport flow rate of the core sampling apparatus was adjusted using a mass flow controller (Sevenstar Inc., D07-9E).

All the connections and tubing were made of stainless steel to avoid potential particle losses due to unexpected bending. The sampling efficiencies of the tested core sampling apparatus, $\eta_{\text {sam }}=N_{2} / N_{1}$, at various tube lengths, particle diameters, and transport flows were investigated. The particle diameter classified by the half-mini DMA was calibrated using standard tetra-heptyl ammonium ions (Ude and Fernández de la Mora 2005). The flow rates of MFCs and aerosol electrometers were calibrated using a bubble flow meter (Sensidyne Inc., Gilibrator 2) before the experiment.

The core sampling apparatus consists of a $46 \mathrm{~cm}$ (or $66 \mathrm{~cm}$ for a larger $\mu$ value) long $3 / 8$ inch outer tube, a $12 \mathrm{~cm}$ long $1 / 4$ inch inner tube, and a reducing tee (Swagelok ${ }^{\mathrm{TM}}$, 
SS-600-3-4-6BT). The core sampling tube protrudes $8 \mathrm{~cm}$ from the midpoint of the tee. The design of protrusion from the tee and tapered inlet in the core sampling tube is to reduce flow disturbance. Reynolds number in both the outer tube and the core sampling tube were kept below 2000 and air flow was assumed to be a fully developed laminar flow as confirmed by numerical simulations using COMSOL Multiphysics.

In addition to the core sampling apparatus, the sampling efficiencies of a tee (Swagelok ${ }^{\mathrm{TM}}$, SS-600-3), a Y fitting (ASOH, HY-3007), and a cross fitting (Swagelok ${ }^{\mathrm{TM}}$, SS-600-4) were also characterized. Flow configurations of all the tested parts are illustrated in Figure 3(b). The length of other tube fittings used between the tested part and the homemade splitter, including a reducing port connector (Swagelok $^{\mathrm{TM}}$, SS-601-PC-4) or a reducing union (Swagelok ${ }^{\mathrm{TM}}$, SS-600-6-4), were also included in the tube length $L$. The tube length between the tested part and the downstream aerosol electrometer was the same to ensure the comparison of measured sampling efficiencies for various tested parts.

\section{Results and discussion}

\subsection{Parameters governing the sampling efficiency}

The sampling efficiency $\eta_{\text {sam }}$ of a core sampling apparatus is governed by two parameters, i.e., the dimensionless loss parameter $\mu$ and the transport-to-sample flow ratio $Q_{t}: Q_{s}$ (as revealed by Eq. 6 and Figure 4). The sampling efficiency increases with an increase in the transport-to-sample flow ratio and/or a decrease in the loss parameter until it reaches the maximum of unity (Figure 4). As shown in Eq. 7 and Figure 2, an increase in the transport-to-sample flow ratio leads to a decrease in the nondimensionalized integration radius $\tilde{r}_{a}$ such that the sampling is more confined around the centerline of the flow, and thus an increase in the sampling efficiency until it reaches the maximum. Significant improvement in the sampling efficiency can be achieved by increasing the transport-to-sample flow ratio when it is low, e.g., increasing from 0 to 0.5 as shown in Figure 4. However, this improvement (without adjusting the loss parameter) is limited when the ratio is high. At a fixed transport-to-sample flow ratio, a decrease in the loss parameter leads to an increase in the concentration of sampled species (Figure 4), and thus higher sampling efficiency until it reaches the maximum of unity. The definition of the loss parameter (Eq. 3) shows that lower diffusivity of the sampled species, shorter tube length, and higher total flow rate result in lower values for this parameter. Note that a change in the transport-to-sample flow ratio may lead to a change in the loss parameter as well, and vice versa.

As shown in Figure 5, experimental results confirm that the sampling efficiency of a 
core sampling apparatus is governed by the two parameters and the trends are the same to those from theoretical analysis. For instance, the sampling efficiency increases with an increase in the transport-to-sample flow ratio (while taking experimental uncertainties into considerations). It also increases with a decrease in the loss parameter until it reaches the maximum of unity. The measured sampling efficiencies agree well with theoretically estimated ones when the loss parameter is less than 0.1 , e.g., their relative deviations are within $5 \%$.

When the loss parameter becomes higher (e.g., 0.1-0.4), the relative deviations between measured and theoretically estimated ones become higher, but still within $44 \%$. Experimental uncertainties contribute to these deviations. Please note that experimentally generating various combinations of the loss parameter and the transportto-sample flow ratio is challenging for a given experimental setup. As shown by the definition of the loss parameter (Eq. 3), its accuracy, especially at higher values, is affected by precision of controlling low flow rate and the accuracy in diffusivity for highly diffusive species (often calculated from Millikan equation and Stokes-Einstein equation using electric mobility diameter). On the other hand, generating conditions with a very small value for the loss parameter (e.g., below 0.05), is also challenge due to the required laminar flow conditions in the core sampling apparatus which adds an upper limit on the total flow rate and difficulties in generating sufficient large particles $(>\sim 5 \mathrm{~nm})$ while operating the wire generator at the flow rate of 20 LPM.

As shown in Figures $4 \& 5$, the loss parameter less than 0.1 is recommended when applying the core sampling method. When the value for the loss parameter is low and a reasonable transport-to-sample flow ratio is used, the sampling efficiency is equal or close to unity. Under such a high sampling efficiency, theoretical and experimental results agree well with each other. It is also relatively insensitive to variations in sampling conditions such that there is less uncertain when using the sampling efficiency for data inversion. In comparison, values for the loss parameter were estimated to be 0.03-0.45 for reported gaseous sulfuric acid measurement (e.g., Brus et al. 2017; Jokinen et al. 2012) and sub-3 nm particles measurement (e.g., Jiang et al. 2011b; Kangasluoma et al. 2016). Their sampling efficiencies can be further improved by optimizing the loss parameter and the transport-to-sample flow ratio using the findings reported here. The uncertainties in sampling efficiencies can also be reduced.

\subsection{Designing and optimizing a core sampling apparatus}

When designing a core sampling apparatus, the transport flow rate $Q_{t}$ is often the only variable to be tuned such that its performance will be optimized as recommended above. This is because that the sample flow rate $Q_{s}$, the tube length $L$, and the diffusivity of 
sampled species $D$ are often known and fixed for a given sampling scenario. The diameter of the tube depends on its availability and is also often fixed. To guide the design for this scenario, Figure 6 illustrates the sampling efficiency as a function of the transport-to-sample flow ratio at various $\mu_{0}$ values (similar to the dimensionless loss parameter $\mu, \mu_{0}$ was defined for the scenario without the transport flow (Eq. 4)). When the transport flow is added and increases while $Q_{s}, L$, and $D$ are the same, the loss parameter $\mu$ will decrease and the sampling efficiency will increase following the line of the same $\mu_{0}$ value. Therefore, the transport flow can be chosen based on the diameter of the available tube while keeping it at the laminar flow condition (Reynolds number $<2000$ ). The sampling efficiency can then be estimated using lines in Figure 6 after the transport flow is set. In most scenarios, it can reach the maximum value of unity.

A core sampling apparatus was designed and tested as an example. It consists of a $3 / 8$ inch outer tube and a 1/4 inch tapered inner tube. The tube length $L$ is $0.46 \mathrm{~m}$. Note that it is also the apparatus used for obtaining data shown in Figure 5. If $1.2 \mathrm{~nm}$ particles is sampled at the sample flow rate $Q_{s}$ of $0.5 \mathrm{LPM}$. The parameter $\mu_{0}$ was estimated to be 0.6. For the $3 / 8$ inch outer tube, a Reynolds number of 2000 corresponds to the total flow rate of 10.8 LPM. Following the line for $\mu_{0}=0.6$ in Figure 6, when the transportto-sample flow ratio is as high as 10 (the transport flow is 5 LPM), the sampling efficiency is approximately unity while the total flow rate (5.5 LPM) is still significantly less than 10.8 LPM. A transport flow of 5 LPM was thus adopted and the corresponding loss parameter $\mu$ is 0.06 , i.e., less than the recommended value of 0.1 . Note that when sampling particles larger than $1.2 \mathrm{~nm}$ (lower diffusivity) using the same sampling conditions, the sampling efficiency will remain to be unity as shown in Figures $4 \& 5$.

As shown in Figure 7(a), experimental results confirm that the sampling efficiency of the designed core sampling apparatus is unity for 1.2-3 nm particles with the transport flow of 5 LPM. At the same conditions (flow rates and the tube length), its sampling efficiencies are significantly higher than those of conventional connectors, i.e., a tee, a cross fitting, and a Y fitting. For instance, the sampling efficiency for $1.2 \mathrm{~nm}$ particles using the core sampling apparatus is $\sim 55 \%$ higher than those of the tee and the cross fitting, and is $\sim 150 \%$ higher than that of the $\mathrm{Y}$ fitting. Similar to the reported deposition of $1 \mathrm{~nm}$ particles at the bifurcating points in tracheae (Shi et al. 2004), additional losses may also occur at the branching points of the tee and the $Y$ fitting. Sudden disturbance of flow field can lead to secondary flow (Tsai and Pui 1990) and thus the redistribution of highly diffusive species. Accordingly, high concentration gradient can occur near tube wall and accelerates diffusional deposition. Since neither the sample flow nor the transport flow experience significant turns near the A-A profile in the core sampling system, additional losses caused by flow splitting is minimized. Even without the 
transport flow, the sampling efficiency of the core sampling apparatus is higher than those of the cross fitting and the tee, and followed by the $Y$ fitting (Figure 7(b)).

Several points should be emphasized when using the core sampling method. Firstly, both the total flow and the sample flow should be kept at laminar conditions. Reynolds number of 2000 is usually used as a criterion, above which laminar flows turn into transitional flows and then turbulent flows. The value of 2000 is not absolute, and it can be smaller in practical use. Secondly, large bends (Wang et al. 2002) need to be avoided for the upstream of the core sampling tube to ensure axisymmetry. Thirdly, the sampled species is assumed to be uniformly distributed at the entrance of a core sampling apparatus. Sampling from flowing gas streams may violate this assumption. Fourthly, the diffusivity of molecules or clusters is likely to be influenced by environmental conditions such as temperature and relative humidity (Brus et al. 2017; Fuller et al. 1966). If so, it should be taken into consideration when estimating the loss parameter and the sampling efficiency.

\section{Conclusions}

Through theoretical and experimental analysis, we quantitatively show that the core sampling method can significantly reduce diffusional losses of diffusive species (e.g., aerosol nanoparticles, ions, and gases) in the sampling line. A maximum sampling efficiency of unity can be readily achieved. By solving the convection diffusion equation with several assumptions, an analytical solution was developed for quantifying the sampling efficiency. Verified using experiments, the developed solution for the core sampling system can be used for revealing its governing parameters, designing an apparatus, and optimizing its performance. The sampling efficiency is governed by a dimensionless loss parameter and the transport-to-sample flow ratio. The core sampling method is recommended to work at the loss parameter less than $0.1 \mathrm{such}$ that its sampling efficiency is equal or close to unity and is also insensitive to variations in sampling conditions. Guided by the analytical solution, a core sampling apparatus was designed and experimentally evaluated. As expected, its sampling efficiency was measured to be approximately unity for $1.2-3 \mathrm{~nm}$ particles, significantly higher than those of the conventional connectors (e.g., a tee, a cross fitting, and a Y fitting) at the same sampling conditions.

\section{Acknowledgement}

Financial support from National Key R\&D Program of China (2017YFC0209503) and the National Natural Science Foundation of China (21876094) are acknowledged. The authors thank Zenghui Miao and Jinghui Zhang for helps in theoretical analysis of the 
core sampling method.

\section{Reference}

Alonso, M., Kousaka, Y., Hashimoto, T., Hashimoto, N. (1997). Penetration of Nanometer-Sized Aerosol Particles Through Wire Screen and Laminar Flow Tube. Aerosol Science and Technology, 27:471-480.

Brus, D., Škrabalová, L., Herrmann, E., Olenius, T., Trávničková, T., Makkonen, U., Merikanto, J. (2017). Temperature-Dependent Diffusion of $\mathrm{H}_{2} \mathrm{SO}_{4}$ in Air at Atmospherically Relevant Conditions: Laboratory Measurements Using Laminar Flow Technique. Atmosphere, 8:132.

Eckert, E. R. G. and Drake, R. M. (1987). Analysis of heat and mass transfer.

Eisele, F. L. and Tanner, D. J. (1993). Measurement of the gas phase concentration of $\mathrm{H}_{2} \mathrm{SO}_{4}$ and methane sulfonic acid and estimates of $\mathrm{H}_{2} \mathrm{SO}_{4}$ production and loss in the atmosphere. Journal of Geophysical Research: Atmospheres, 98:90019010 .

Fernández de la Mora, J. and Kozlowski, J. (2013). Hand-held differential mobility analyzers of high resolution for 1-30 nm particles: Design and fabrication considerations. Journal of Aerosol Science, 57:45-53.

Fuller, E. N., Schettler, P. D., Giddings, J. C. (1966). New method for prediction of binary gas-phase diffusion coefficients. Industrial \& Engineering Chemistry, 58:18-27.

Gormley, P. and Kennedy, M. (1948). Diffusion from a stream flowing through a cylindrical tube, Proceedings of the Royal Irish Academy. Section A: Mathematical and Physical Sciences, 52:163-169.

Jiang, J., Chen, M., Kuang, C., Attoui, M., McMurry, P. H. (2011a). Electrical Mobility Spectrometer Using a Diethylene Glycol Condensation Particle Counter for Measurement of Aerosol Size Distributions Down to $1 \mathrm{~nm}$. Aerosol Science and Technology, 45:510-521.

Jiang, J., Zhao, J., Chen, M., Eisele, F. L., Scheckman, J., Williams, B. J., Kuang, C., McMurry, P. H. (2011b). First Measurements of Neutral Atmospheric Cluster and 1-2 nm Particle Number Size Distributions During Nucleation Events. Aerosol Science and Technology, 45:ii-v.

Jokinen, T., Sipilä, M., Junninen, H., Ehn, M., Lönn, G., Hakala, J., Petäjä, T., Mauldin Iii, R. L., Kulmala, M., Worsnop, D. R. (2012). Atmospheric sulphuric acid and neutral cluster measurements using CI-APi-TOF. Atmospheric Chemistry and Physics, 12:4117-4125.

Kangasluoma, J., Attoui, M., Junninen, H., Lehtipalo, K., Samodurov, A., Korhonen, F., Sarnela, N., Schmidt-Ott, A., Worsnop, D., Kulmala, M., Petäjä, T. (2015). Sizing of neutral sub 3nm tungsten oxide clusters using Airmodus Particle Size Magnifier. Journal of Aerosol Science, 87:53-62.

Kangasluoma, J., Franchin, A., Duplissy, J., Ahonen, L., Korhonen, F., Attoui, M., Mikkilä, J., Lehtipalo, K., Vanhanen, J., Kulmala, M., Petäjä, T. (2016). Operation of the Airmodus A11 nano Condensation Nucleus Counter at various inlet pressures and various operation temperatures, and design of a new inlet 
system. Atmospheric Measurement Techniques, 9:2977-2988.

Knopf, D. A., Poschl, U., Shiraiwa, M. (2015). Radial diffusion and penetration of gas molecules and aerosol particles through laminar flow reactors, denuders, and sampling tubes. Analytical Chemistry, 87:3746-3754.

Kundu, P., Cohen, I., Dowling, D. (2012). Chapter 12-turbulence. Fluid Mechanics (Fifth Edition), Academic Press, Boston:541-620.

Mikhailov, M. D. and Cotta, R. M. (1997). Eigenvalues for the Graetz problem in slipflow. International Communications in Heat and Mass Transfer, 24:449-451.

Sellars, J. R., Tribus, M., Klein, J. (1954). Heat transfer to laminar flow in a round tube or flat conduit: the Graetz probem extended. WADC Technical Report, 54.

Shi, H., Kleinstreuer, C., Zhang, Z., Kim, C. S. (2004). Nanoparticle transport and deposition in bifurcating tubes with different inlet conditions. Physics of Fluids, 16:2199-2213.

Stolzenburg, M. R. and McMurry, P. H. (1991). An Ultrafine Aerosol Condensation Nucleus Counter. Aerosol Science and Technology, 14:48-65.

Subramanian, S. R. (2012). The Graetz Problem.

Tsai, C.-J. and Pui, D. Y. H. (1990). Numerical Study of Particle Deposition in Bends of a Circular Cross-Section-Laminar Flow Regime. Aerosol Science and Technology, 12:813-831.

Ude, S. and Fernández de la Mora, J. (2005). Molecular monodisperse mobility and mass standards from electrosprays of tetra-alkyl ammonium halides. Journal of Aerosol Science, 36:1224-1237.

Wang, J., Flagan, R. C., Seinfeld, J. H. (2002). Diffusional losses in particle sampling systems containing bends and elbows. Journal of Aerosol Science, 33:843-857.

Yook, S.-J. and Pui, D. Y. H. (2006). Experimental Study of Nanoparticle Penetration Efficiency Through Coils of Circular Cross-Sections. Aerosol Science and Technology, 40:456-462. 


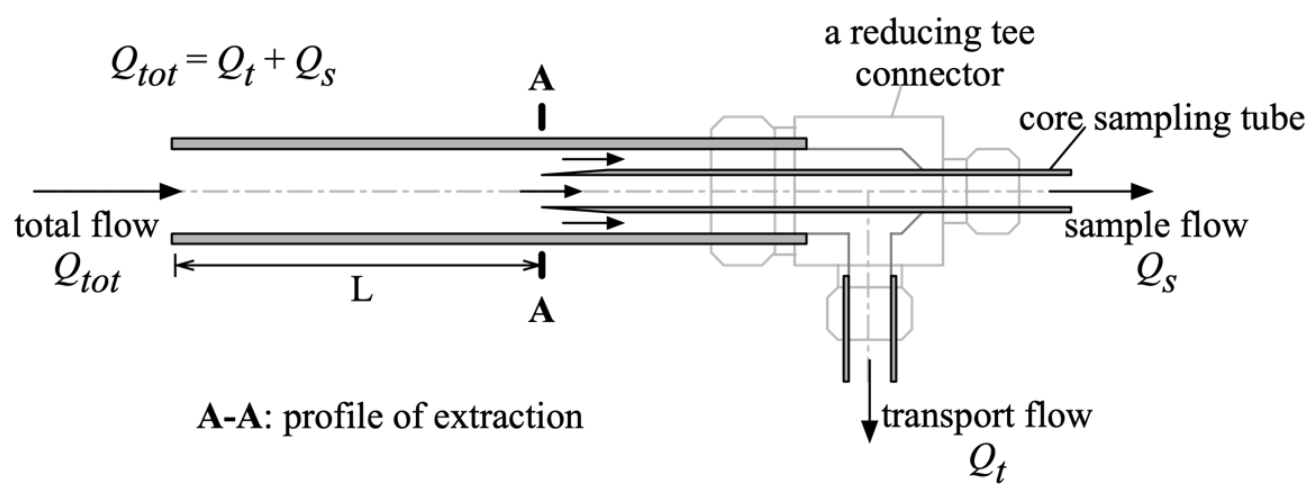

Figure 1 Schematic of a core sampling apparatus and its parameters 


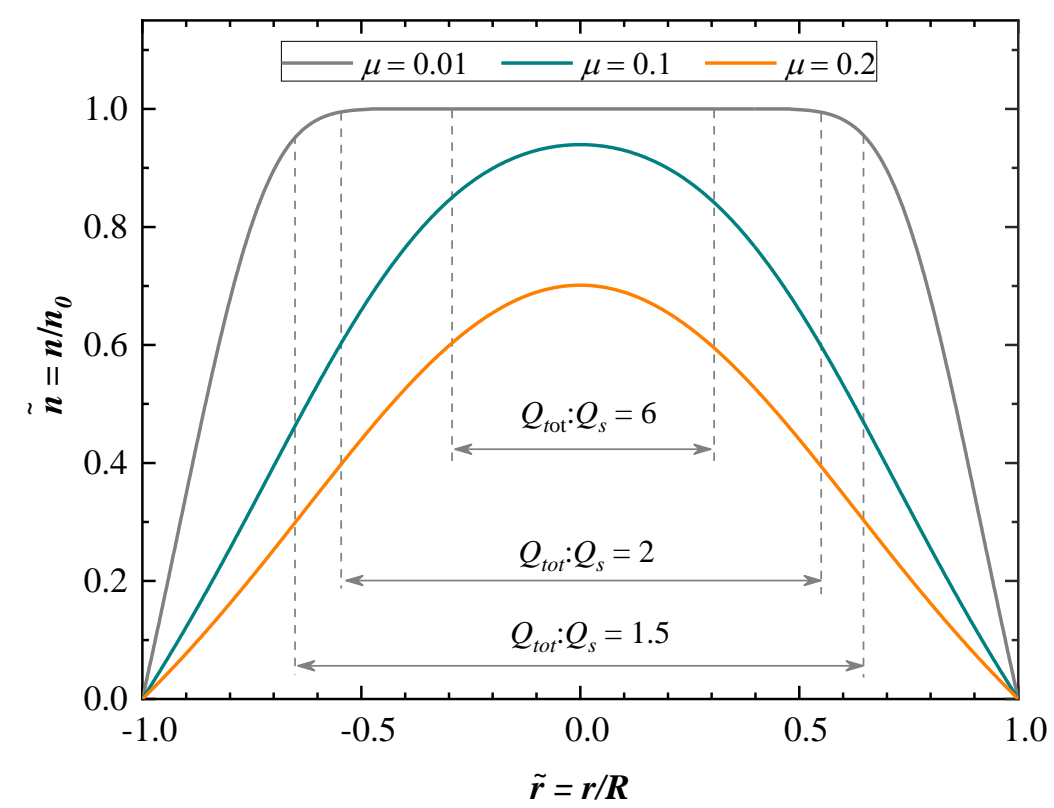

Figure 2 The number concentration distributions of diffusive species at the A-A profile for various $\mu$ values. Between each pair of vertical dashed lines is the core region at different value for the $Q_{t o t}: Q_{s}$ ratio, i.e., the range of integration (corresponding to $\tilde{r}_{a}$ in Eq. 6) for calculating the sampling efficiency. Note that $Q_{t o t}: Q_{s}$ is related to the transport-to-sample flow ratio $Q_{t} / Q_{s}$, i.e., $Q_{t o t}: Q_{s}=Q_{t} / Q_{s}+1$. 
(a)
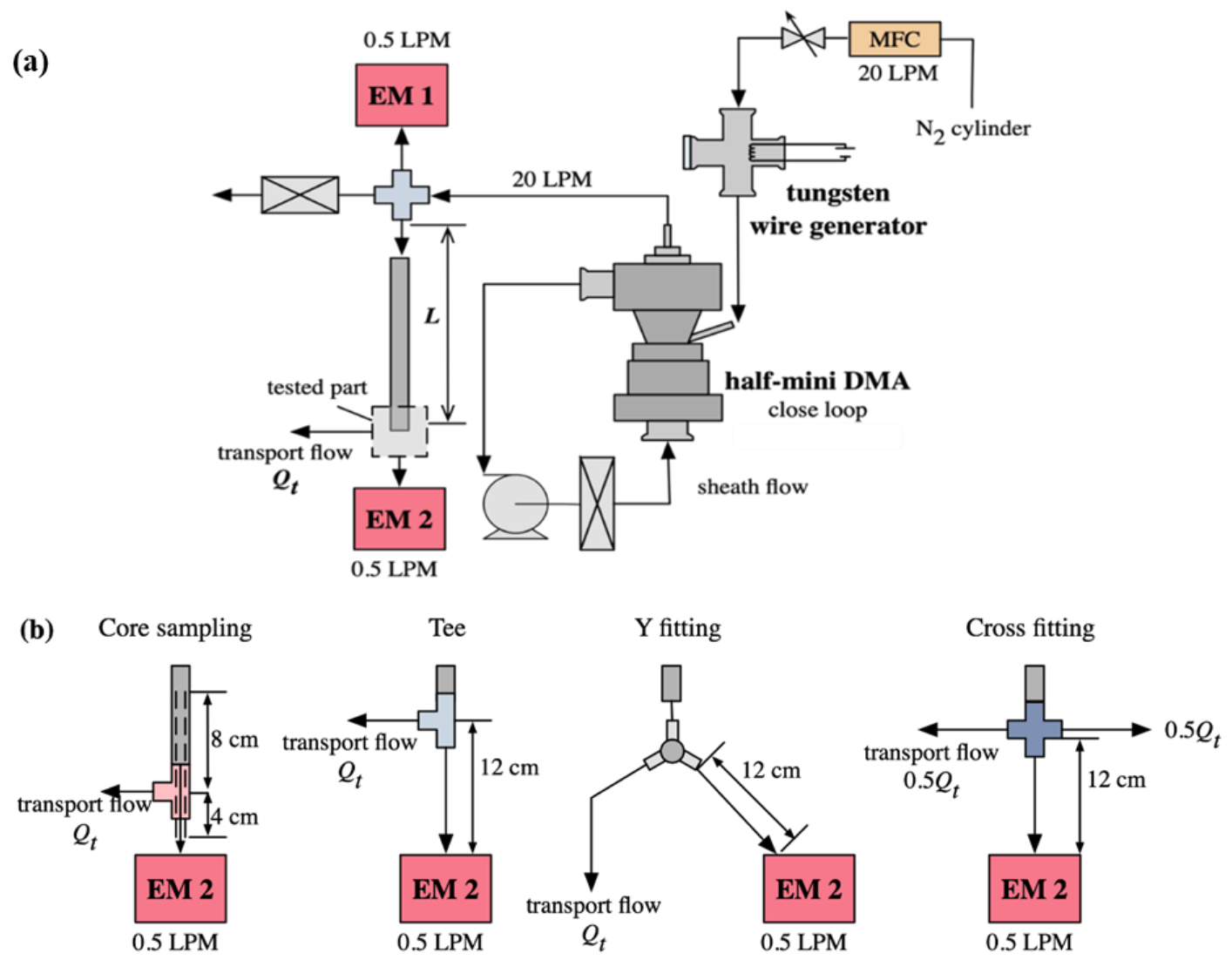

Figure 3 (a) Schematic of the experimental setup to calibrate the sampling efficiency using 1-5 nm tungsten oxide nanoparticles. The tested part can be a core sampling apparatus, a tee, a Y fitting, or a cross fitting. (b) Detailed schematics of how flow splits in various tested parts. The $12 \mathrm{~cm}$ length in the upstream of EM 2 as shown in (b) is not included in the tube length $L$ as shown in (a). Note that EM denotes an aerosol electrometer; MFC denotes a mass flow controller; and DMA denotes a differential mobility analyzer. 


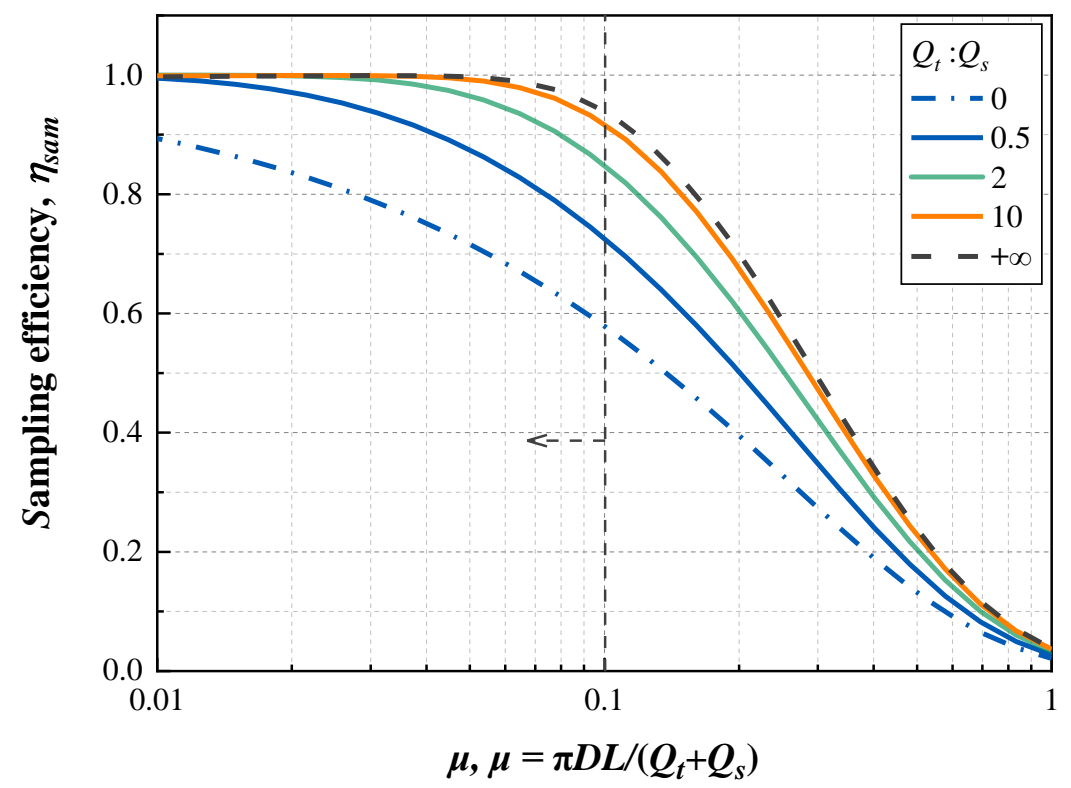

Figure 4 The sampling efficiency of a core sampling system as a function of the dimensionless loss parameter $\mu$ at various transport-to-sample flow ratios $(T=293 \mathrm{~K}, P=1 \mathrm{~atm})$. Note that the curve of zero transport flow agrees with the results predicted by the Gormley-Kennedy formula (Gormley \& Kennedy, 1948). The loss parameter less than 0.1 is recommended when using the core sampling method. 


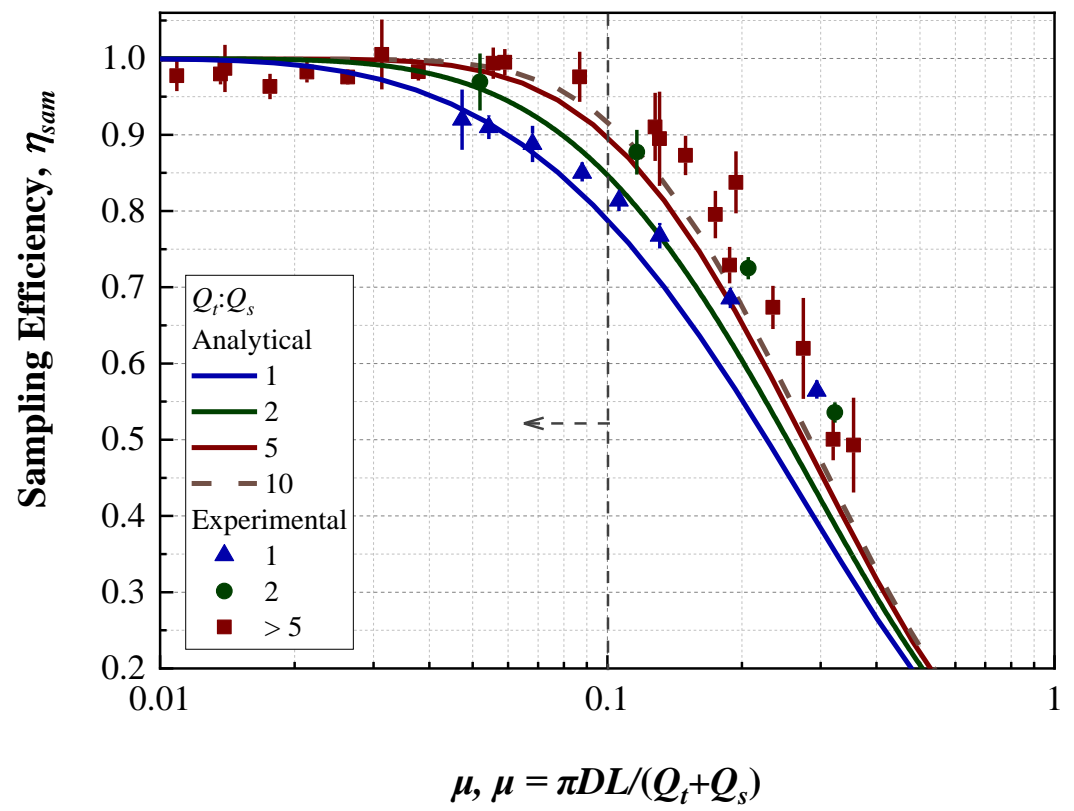

Figure 5 The sampling efficiency of a core sampling system under various sampling conditions. The error bar represents standard deviation of the measured sampling efficiency. The tube length $L$ is $46 \mathrm{~cm}$ or 66 $\mathrm{cm}$. The sample flow rate $Q_{s}$ is mostly $0.5 \mathrm{LPM}$ (a few of data points were obtained at $0.3 \mathrm{LPM}$ to achieve higher values for the loss parameter $\mu$ ). $\mu$ was varied by changing $L, Q_{s}, Q_{t}$, and $d_{p}$. Maximum value of $Q_{i}: Q_{s}$ used in the experiment was 10 . The loss parameter less than 0.1 is recommended when using the core sampling method 


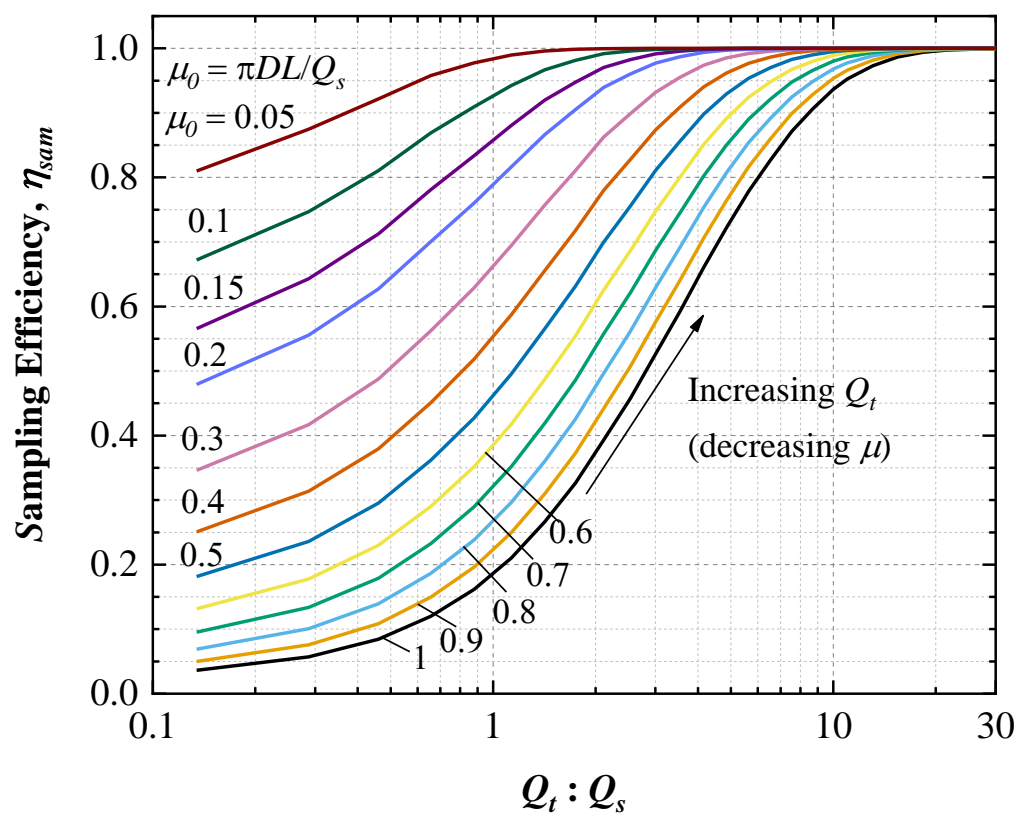

Figure 6 The sampling efficiency of a core sampling system as a function of the transport-to-sample flow ratio at various $\mu_{0}$ values. Similar to the dimensionless loss parameter $\mu_{,} \mu_{0}$ was defined for the sampling scenario without the transport flow. Its values for various conditions are presented next to the corresponding curves. For each curve (the same $\mu_{0}$ ), the diffusivity $D$, the tube length $L$, and the sample flow rate $Q_{s}$ are fixed and the transport flow rate $Q_{t}$ is varied which is often the case when applying the core sampling system. 


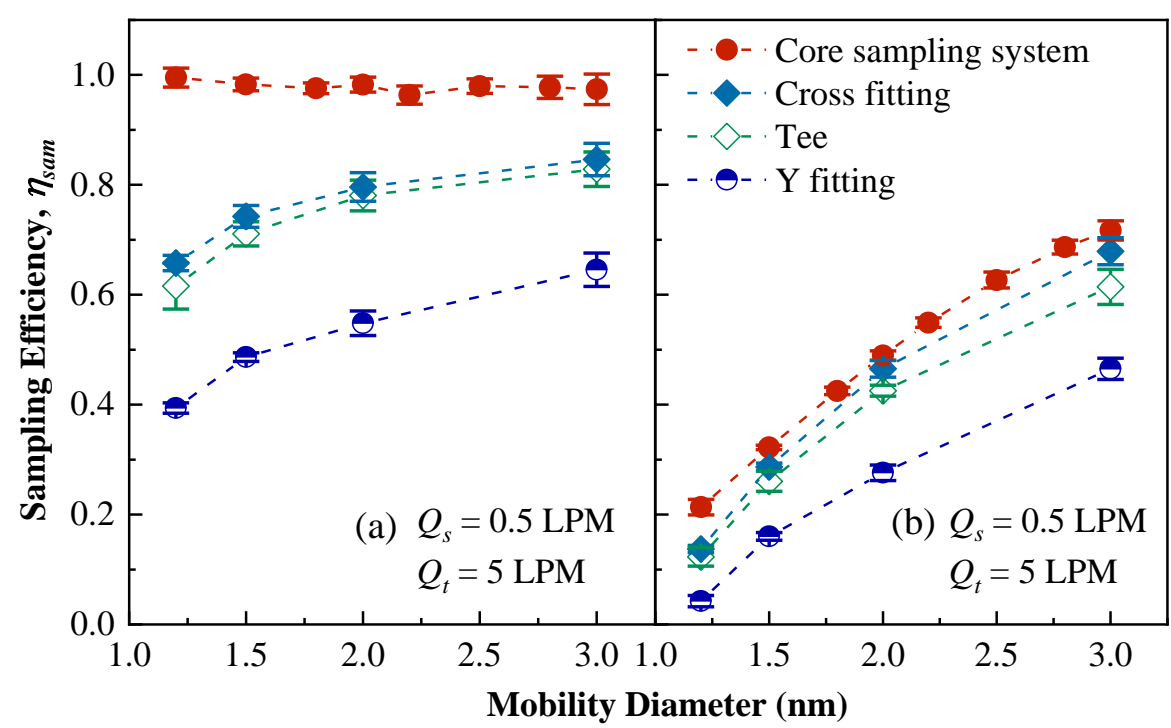

Figure 7 The sampling efficiencies of a core sampling apparatus and conventionally used connectors (a tee, a cross fitting, and a $\mathrm{Y}$ fitting): (a) when the transport-to-sample flow ratio $Q_{t}: Q_{s}$ is 10 and (b) without the transport flow. The tube length $L$ is $46 \mathrm{~cm}$. The error bars indicate standard deviation of the measured efficiencies. 


\title{
Supplementary Information
}

\section{Theoretical and experimental analysis of the core sampling method: reducing diffusional losses in aerosol sampling line}

\author{
Yueyun $\mathrm{Fu}^{1 \dagger}$, Mo Xue ${ }^{1 \dagger}$, Runlong $\mathrm{Cai}^{1}$, Juha Kangasluoma ${ }^{2,3}$, and Jingkun Jiang ${ }^{1 *}$ \\ ${ }^{1}$ State Key Joint Laboratory of Environment Simulation and Pollution Control, \\ School of Environment, Tsinghua University, 100084 Beijing, China \\ 2 Aerosol and Haze Laboratory, Advanced Innovation Center for Soft Matter Science \\ and Engineering, Beijing University of Chemical Technology, Beijing, China \\ ${ }^{3}$ Institute for Atmospheric and Earth System Research / Physics Faculty of Science, \\ University of Helsinki, P.O. Box 64, 00014 Helsinki, Finland \\ $\dagger$ : Yueyun Fu and Mo Xue contributed equally to this study. \\ *Correspondence to: J. Jiang (jiangjk@tsinghua.edu.cn)
}

\section{(1) Derive the analytical solution}

Assuming that there are no external forces causing directional movement of the diffusive species, the spatial distribution of these diffusive species upstream of the AA profile follows a steady-state convection diffusion equation (CDE):

$$
D \cdot \nabla^{2} n^{\prime}-u^{\prime} \cdot \nabla n^{\prime}=0
$$

where $n$ ' is the concentration of diffusive species as a function of the spatial position in a cylindrical coordinate, $n(r, \theta, z) ; D$ is the diffusivity of the sampled diffusive species; $u^{\prime}$ is the fluid speed which is a vector function of spatial position, $u^{\prime}(r, \theta, z) ; \nabla$ is the nabla operator; $\nabla n^{\prime}$ is the gradient of $n^{\prime}(r, \theta, z)$; and $\nabla^{2}$ is the Laplace operator. According to symmetry, $u$ ' and $n$ ' is independent of the angle $\theta$. When Assumptions $1 \sim 2$ are valid, convection diffusion can be reformulated into the Graetz problem: 
- Assumption 1. The flow in the cylindrical tube is a fully developed laminar flow.

- Assumption 2. The diffusion in axial direction is negligible compared to the axial convection.

Reynold number $\left(\operatorname{Re}=u_{a v g} d / v\right)$, where $d$ is the tube diameter and $v$ is the kinematic viscosity of fluid, is a commonly used criterion to distinguish laminar flow $(\operatorname{Re}<2000)$ from turbulent flow (Kundu et al. 2012). When sampling from static fluid, Assumption 1 is invalid within the entrance length near the entrance where the fluid develops from plug flow to Hagen-Poiseuille flow. The entrance length for establishing a parabolic velocity profile from a uniform velocity profile, where $L_{f d}=0.05 d \operatorname{Re}$ (Bergman et al. 2011), is relatively short compared to the conventionally used tubing for sampling (e.g., $L_{f d}=0.54 \mathrm{~m}$ and $\operatorname{Re}=1390$ when sampling 7.5 LPM through a $3 / 8$ inch tube). For the case of a shorter tube using higher flow rate, entrance length is not negligible. Such a case is discussed in section (2).

Peclèt number $\left(\mathrm{Pe}=u_{\text {avg }} d / D\right)$ describes the relative transmission efficiency of convection to that of diffusion. Assumption 2 is valid for $\mathrm{Pe}>100$ and for $1<\mathrm{Pe}<100$ when the tube length $L>2.5 d$ (Tan and Hsu 1971). When sampling species with a diffusion coefficient of $10^{-5} \mathrm{~m}^{2} \mathrm{~s}^{-1}$ at a flow rate of $1 \mathrm{LPM}$ using a $1 / 4$ inch tube, $\mathrm{Pe}=$ 232. Therefore, Assumption 2 is valid in typical core sampling applications.

Based on symmetry and Assumptions 1 2, the expanded expression of Eq. S1 is reformulated into the Graetz problem as:

$$
2 u_{\text {avg }} \cdot\left[1-(r / R)^{2}\right] \cdot \frac{\partial n(r, z)}{\partial z}=D \cdot \frac{1}{r} \cdot \frac{\partial}{\partial r}\left(r \frac{\partial n(r, z)}{\partial r}\right)
$$

where $r$ and $z$ are radial and axial coordinates; $u_{\text {avg }}$ is the average velocity of fluid; $R$ is the outer tube radius; and $n(r, z)$ is the concentration as a function of $r$ and $z$. Eq. S2 is an expression of the dimensional Graetz problem. Letting $n_{0}$ be the uniform concentration of the diffusive species at the entrance of the tubing, the boundary conditions of Eq. S2 are:

- Condition 1. $n=n_{0}$ at $z=0$

- Condition 2. $n=0$ at $r=R$

- Condition 3. $\partial n / \partial r=0$ at $r=0$

Condition 1 means uniform concentration at $z=0$. It is invalid in the tubing downstream 
of fittings due to the evolvement of concentration profile. Condition 2 means complete losses of species onto tube wall. Therefore, nanoparticles and molecular clusters are eligible for Condition 2, while some gaseous species (e.g., nitric acid and $\mathrm{NO}_{\mathrm{x}}$ ) may be not. Condition 3 indicates symmetry along the axial direction. With Conditions 1 3, Gormley and Kennedy (1948) solved the average sampling efficiency at a cross section from the Graetz problem. Experiments proved that Assumptions 1 2 and conditions $1 \sim 3$ are valid for the sampling efficiency of particles down to $2 \mathrm{~nm}$ (Alonso et al. 1997).

The concentration distribution $\tilde{n}(\tilde{r}, \tilde{z})$ is obtained with conditions $1 \sim 3$ from the Graetz problem after non-dimensionalization. The sampling efficiency of a core sampling method can be calculated by integrating $\tilde{n}$ with respect to $\tilde{r}$ when Assumption 3 is valid.

- Assumption 3. When the total flow splits into the sample flow and the transport flow at the A-A profile, re-establishment of flow field takes place within negligible tube length.

Assumption 3 indicates that the sample flow and the transport flow split immediately. Actually, the laminar total flow smoothly develops into two laminar flows (the sample flow in the core sampling tube and the transport flow in the annular region between the core sampling tube and the outer tube) within a relatively short length. The length of unparalleled streamlines upstream of the A-A profile can be defined as flow transforming length $L_{f t}$ (Figure $\mathrm{S} 1(\mathrm{a})$ ). When the point with axial distance of $z$ is within the transforming length $\left(L-L_{f t}<z \leqslant L\right)$, convection diffusion equation cannot be reformulated into the Graetz problem due to existence of the radial convection. The real $n(r, L)$ at the A-A profile is different from that calculated from the Graetz problem. Therefore, there probably exists some differences between sampling efficiencies obtained by integrating calculated concentration at $z=L$ (at the A-A profile) and the real efficiencies. However, $L_{f t}$ is usually short (Figure S3(b)), and Assumption 3 is unviolated when dimensionless Schmidt number $S c>10\left(S_{c}=v / D\right.$, where $v$ is the kinematic viscosity of the fluid) (Chen and Comparin 1976), which is valid for particles larger than $2 \mathrm{~nm}$.

Figure S1(a) further illustrates how the flow transforms, when the $\tilde{r}_{a}$ (related to the transport-to-sample flow ratio shown in Eq. 7) varies. i.e., $\tilde{r}_{a}<R_{\text {core }} ; \tilde{r}_{a}=R_{\text {core }}$; and $\tilde{r}_{a}>R_{\text {core }}$,where $R_{\text {core }}$ is the inner radius of the core sampling tube. As the radial speed, $u_{r}$, exists in the first and the last situation, the radial convection term $u_{r}(r, z) n(r, z)$ in Figure $\mathrm{S} 1(\mathrm{~b})$ is no longer zero. When $\mathrm{Pe}>100$, the axial diffusion term $D \partial n(r, z) / \partial z$ is negligible compared to the axial convection term $u_{z}(r, z) n(r, z)$. Analogously, 
Assumption 3 can be interpreted as that the radial diffusion term $D \partial n(r, z) / \partial r$ is negligible compared to the radial convection term $u_{r}(r, z) n(r, z)$ within the short flow transforming length $L_{f t}$, when $r<R \bullet \tilde{r}_{a}$. i.e., the ratio $u_{r}(r, z) n(r, z) /(D \partial n(r, z) / \partial r)$ is sufficiently large.

(a)
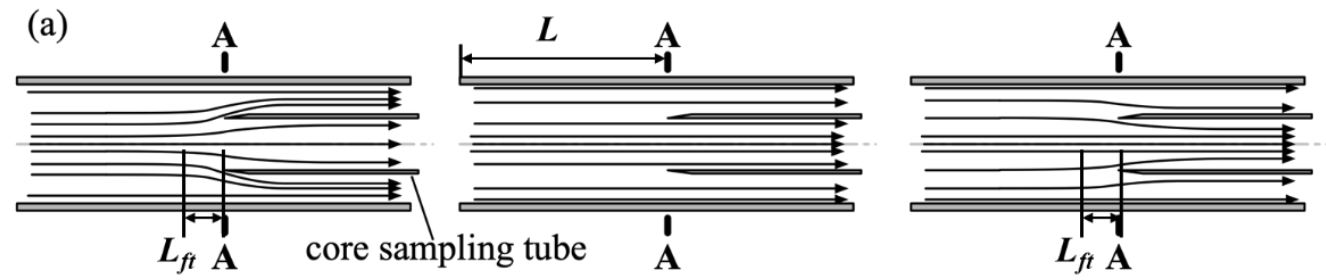

(b)

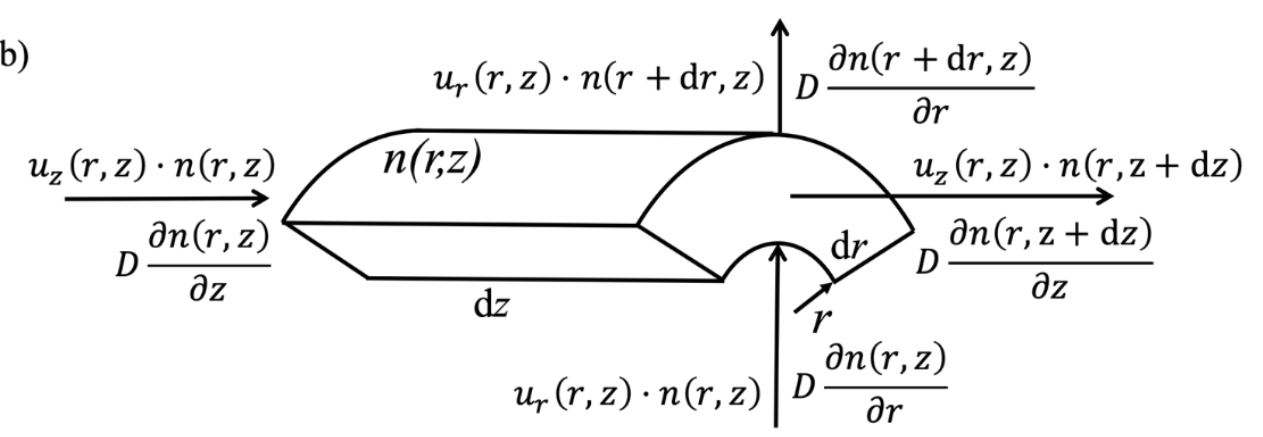

Figure S1 Illustrative schematics of Assumption 3. (a) Schematic diagram of three possible streamline orientation of the laminar total flow near the A-A profile. Schematics from the left to the right respectively correspond to the cases of $\tilde{r}_{a}<R_{\text {core }}, \tilde{r}_{a}=R_{\text {core }}$, and $\tilde{r}_{a}>R_{\text {core }}$, where $R_{\text {core }}$ is the inner radius of the core sampling tube. (b) Various mass flux terms enter or exit an infinitesimal volume in cylindrical coordinate system. The mass flux terms are caused by either convection or diffusion in axial and radial directions.

\section{(2) Comparisons between analytical solutions and numerical simulations}

To test the above assumptions, numerical simulations using COMSOL Multiphysics (COMSOL Inc., Version 5.3, a finite element analysis software package) are conducted and compared with the analytical solution. In COMSOL Multiphysics, convection diffusion equation is numerically solved without Assumption 1-3. In simulation, the total flow is $10.8 \mathrm{LPM}$ in a $1 / 2$ inch tube, and the sample flow is 0.8 LPM in a $1 / 4$ inch core sampling tube. Length $L$ between the entrance and the A-A profile is $0.5 \mathrm{~m}$. The diffusivity is $0.1 \mathrm{~cm}^{2} \mathrm{~s}^{-1}$, temperature is $293 \mathrm{~K}$, ambient pressure is $1 \mathrm{~atm}$, and therefore $\mu$ is approximately $0.087 . \eta_{\text {sam }}$ from the analytical solution is 0.95 , which is $0.6 \%$ lower than that from simulations using COMSOL Multiphysics. According to simulations, the ratio of radial convection term to diffusion term $u_{r}(r, L-\Delta L) n(r, L-\Delta L) /(D \partial n(r, L-\Delta L) / \partial r)$ $=288\left(\Delta L\right.$ is a positive value approximating zero) at $r=R_{\text {core }}$, which indicates a relatively negligible radial diffusion term. $R_{\text {core }}$ is the inner radius of the core sampling 
tube. When $L$ is extended to $1.5 \mathrm{~m}$ and other parameters remain the same, $\mu$ is approximately 0.261 . $\eta_{\text {sam }}$ from the analytical solution is 0.55 . It is $3.4 \%$ higher than the simulations, and $u_{r}(r, L-\Delta L) n(r, L-\Delta L) /(D \partial n(r, L-\Delta L) / \partial r)=674$ at $r=R_{\text {core }}$. As $u_{r}(r$, $L-\Delta L) n(r, L-\Delta L) / D \partial n(r, L-\Delta L) / \partial r \gg 1$ at $r=R_{\text {core }}$ in both the cases, the radial diffusion term is negligible compared to the radial convection term within the flow transforming length $L_{f t}$. This confirms that Assumption 3 is a valid simplification when the total flow splits.
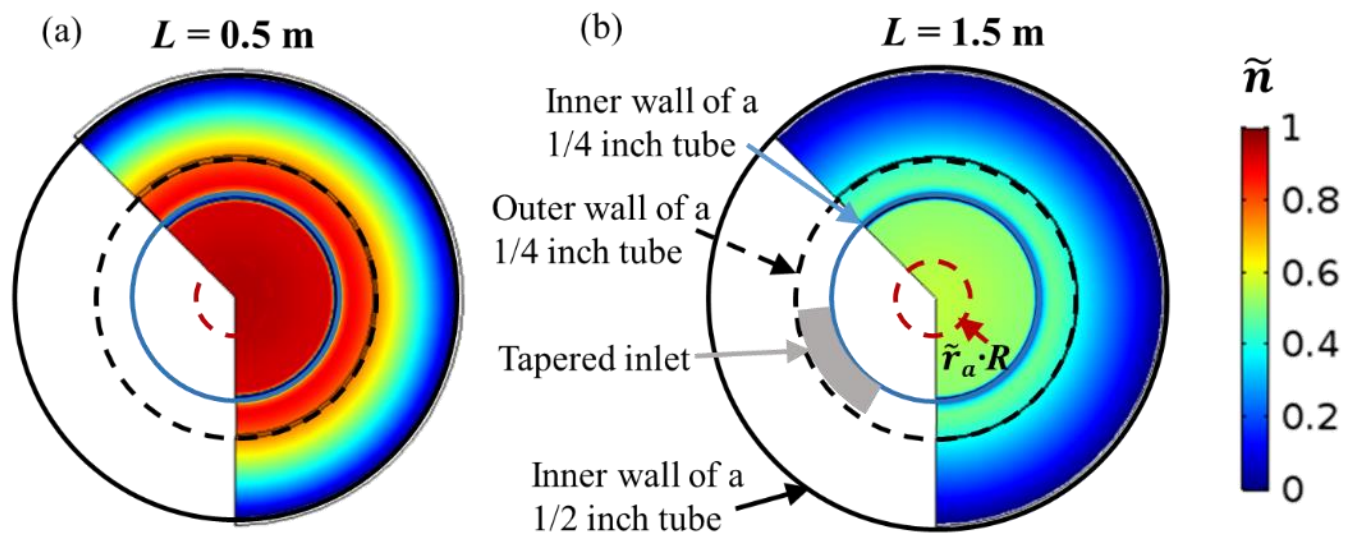

Figure S2 Concentration distribution of the sampled species at the A-A profile simulated using COMSOL Multiphysics: (a) $L$ is $0.5 \mathrm{~m}$, (b) $L$ is $1.5 \mathrm{~m} . \tilde{n}=n / n_{0}$; and $R \cdot \tilde{r}_{a}$ describes where the total flow splits in Assumption 3. The color bar denotes the normalized concentration. The blue solid circle is the inner wall of the core sampling tube, which is the integration range of $\tilde{n}$ with respect to $r$ in simulations to obtain average $\tilde{n}$ at the entrance of the core sampling tube (i.e., sampling efficiency). The dark red region within the blue solid circle in (a) indicates the sampling efficiency closed to the unity, because low concentration of species exits through transport flow.

The laminar flow assumption does not significantly influence the analytical results in the simulated conditions. For the total flow rate of 10.8 LPM in a $1 / 2$ inch tube, Reynolds number $(\mathrm{Re})$ is 1517 and the entrance length $L_{f d}$ is $0.77 \mathrm{~m} . L_{f d}$ is nonnegligible compared to the tube length of $0.5 \mathrm{~m}$ (Figure S3(a)). The core sampling apparatus satisfies the conventional laminar criterion $(\operatorname{Re}<2000)$, however, it violates the Assumption 1 of a fully developed laminar flow, as the total flow is not yet developed at the A-A profile. The analytical sampling efficiency at the A-A profile is only $0.6 \%$ lower than the simulated sampling efficiency, even in this case with relatively short tube length. 

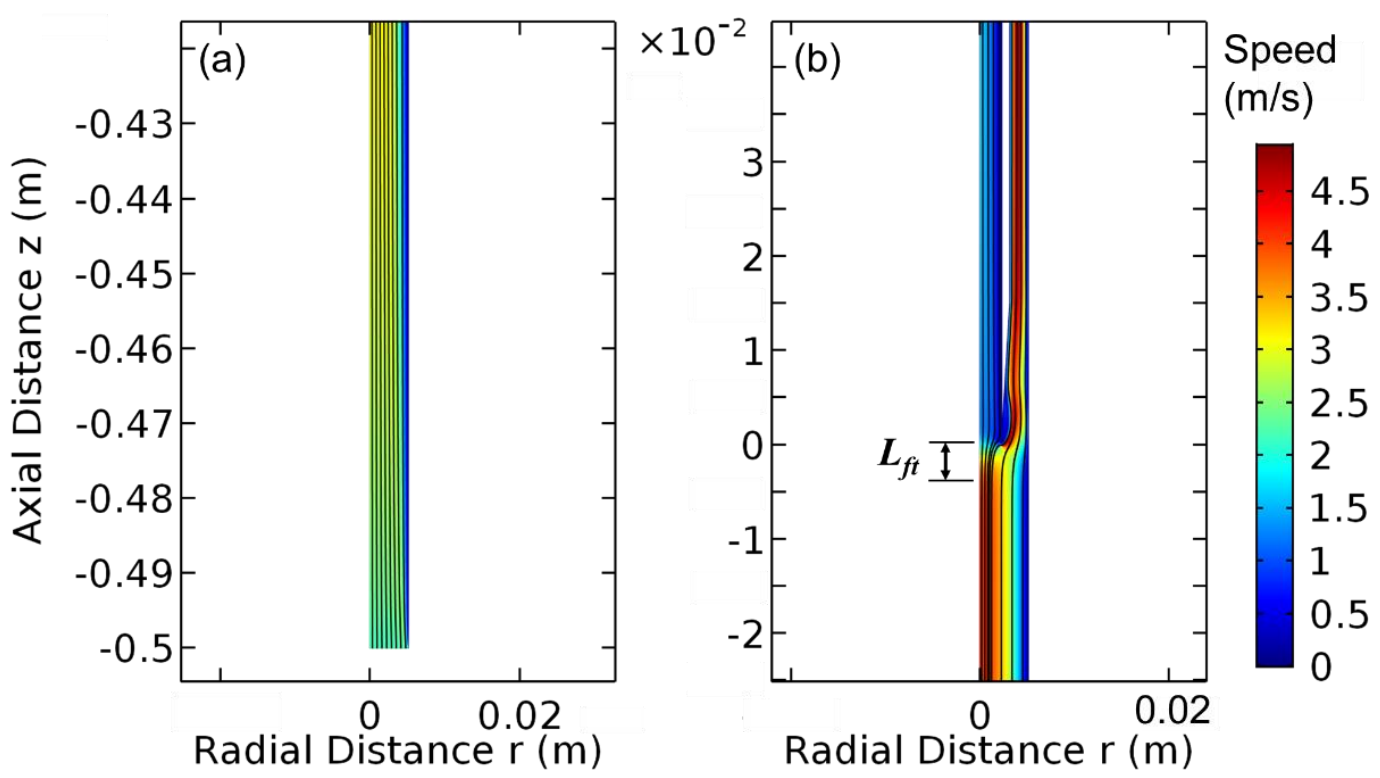

Figure S3 Velocity field and streamlines of simulated results $(L=0.5 \mathrm{~m})$, (a) near the entrance $(z=-0.5$ $\mathrm{m})$ where plug flow gradually transforms into Hagen-Poiseuille flow, (b) near the A-A profile $(z=0 \mathrm{~m})$. The color bar denotes the axial speed of the fluid. The flow is upward. Considering the axisymmetry, only half of the axial sectional profile is presented in the colored area. In (b), the flow transforming length $L_{f t}$ is less than $0.005 \mathrm{~m}$, which is negligible compared to the tube length $(L=0.5 \mathrm{~m})$ and is consistent with Assumption 3.

\section{(3) Explicit illustration of $\lambda_{m}$ and $A_{m}$ in the MATLAB script}

$\lambda_{m}$ are eigenvalues of the Graetz problem. They are zeros of the confluent hypergeometric function $M(1 / 2-\lambda / 4,1, \lambda)$ solved by numerical methods. The multiplicative coefficients $A_{m}$ are determined according to $\lambda_{m}$ (Sellars et al. 1954). When $m$ is infinitely large, there is an asymptotic analytical solution of $\lambda_{m}$, where $\lambda_{m}=$ $4 m+2 / 3$ (Sellars et al. 1954). However, the Graetz problem at $\tilde{z}=0$ is highly sensitive to the digit precision of the predetermined $\lambda_{m}$. Therefore, it is important to use precise and accurate eigenvalues especially when $m$ is small.

In the attached MATLAB (MathWorks Inc., Release 2018b) script, 15 eigenvalues $\lambda_{m}$ are provided, which are obtained from numerical methods of solving the zeros (Mikhailov and Cotta 1997; Wolfram 1991). The corresponding multiplicative coefficient $A_{m}$ in Eq. 2 is determined from the relation given below (Sellars et al. 1954), 


$$
\begin{aligned}
& A_{m}=\frac{\int_{0}^{1} \tilde{r}\left(1-\tilde{r}^{2}\right) \phi_{m}(\tilde{r}) d \tilde{r}}{\int_{0}^{1} \tilde{r}\left(1-\tilde{r}^{2}\right) \phi_{m}{ }^{2}(\tilde{r}) d \tilde{r}}=\frac{-2}{\left.\lambda_{m} \frac{\partial \phi_{m}}{\partial \lambda}\right|_{\substack{\tilde{r}=1 \\
\lambda=\lambda_{m}}},} \\
& \phi_{m}(\tilde{r})=e^{-\lambda_{m} \tilde{r}^{2} / 2} M\left(\frac{1}{2}-\frac{\lambda_{m}}{4}, 1, \lambda_{m} \tilde{r}^{2}\right)
\end{aligned}
$$

\section{(4) A homemade four-way flow splitter}

A homemade four-way flow splitter was designed and used for experiments. The outlet of the half-mini DMA in Figure 3 is a 1/4 inch tube, where a flow rate of 20 LPM has a correspondingly high Reynolds number $(\mathrm{Re}=6270)$. The expanding cone shape in the splitter is designed to gradually reduce Re, which becomes 358 in the cylinder section with a diameter of $40 \mathrm{~mm}$. It is presumed that there is no significant turbulence at the entrances of the two flank outlets, although the fully developed laminar flow is not yet established.

To ensure the comparisons, the average concentration in the two split outflows from a flow splitter should be the same when calibrating the sampling efficiency at various flow rates. In the homemade four-way flow splitter, the two flank outlets directly sample from the core of the inflow. Analogous to the concentration profile shown in Figure S2, the concentration varies less at the core region than near the tube wall. Therefore, the average concentration at the entrances of the two flank outlets is expected to be the same, especially when the sum of two flank outflows is small compared to the total inflow. The concentration ratio of the two outflows was tested to be the unity, when sampling 1-5 nm particles with the inflow of 20 LPM (Figure S4 (b)(c)). The actual flow rates of the two outflows varies from $0.5 \mathrm{LPM} / 0.5 \mathrm{LPM}$ to 0.5 LPM/5.5 LPM in the experiments shown in Figure 3, except for a few cases of 0.3 LPM/0.3 LPM. The tests of the splitter cover the range of actual outflow rates using in the experiments. 


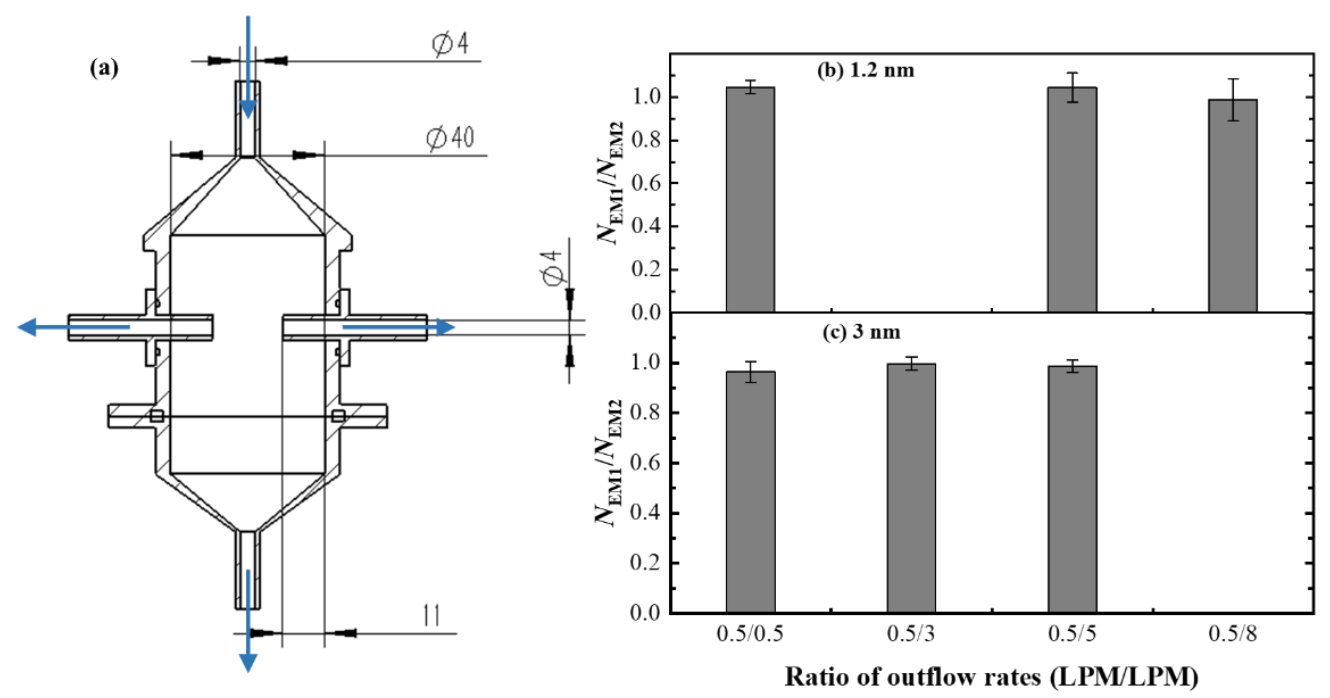

Figure S4 (a) The schematic of a homemade four-way flow splitter. The key dimensions are in unit mm. The blue arrows indicate the inflow and outflows. When used for the experiments shown in Figure 3, the upper inlet is connected the outlet of the half-mini DMA; the flank outlets are connected to aerosol electrometers; and the lower outlet is for the excess flow. (b)(c) The ratio of number concentration of monodisperse tungsten oxide particles measured by two aerosol electrometers (EM 1 and EM 2, TSI Inc., Model 3068B) at various outflow rates ratio. EM 1 and EM 2 are respectively connected to the two flank outlets of the splitter using the same tube length. Sampling flow of EM 1 is 0.5 LPM and sampling flow of EM 2 varies from 0.5 to 8 LPM. The inflow of the splitter is 20 LPM. The error bar denotes the standard deviation of the ratio of the number concentration.

\section{(5) A MATLAB script for calculating the sampling efficiency of a core sampling apparatus}

$\%$ analytical solution for particle diffusion loss when passing through a cylinder tubing

\% Yueyun Fu, on Nov. 13, 2018, at Tsinghua University

$\% \% \% \% \%$

$\%$ Graetz problem

$\% 2\left(1-r^{\wedge} 2\right) * d n / d x=\left(d^{\wedge} 2 n / d r^{\wedge} 2+1 / r^{*} d n / d r\right)$

$\% n(0, r)=n 0$ for $r<1$

$\% n(x, 1)=0$ for $x>0$

$\% \mathrm{dn} / \mathrm{dr}=0$ for $r=0$

$\% \mathrm{n}=0$ for $\mathrm{x}=$ infinite

\% solution: $\mathrm{n}(\mathrm{miu}, \mathrm{r})=\operatorname{sum}\left\{\mathrm{Ai} * \mathrm{M}\left(1 / 2-\right.\right.$ lamda/4,1,lamda*$\left.{ }^{\wedge} 2\right) * \exp \left(-\operatorname{lamda}{ }^{*}{ }^{\wedge} 2 / 2\right) * \exp (-$ miu*lamda^2/2) \}

\% Some inaccuracy occurs when miu approaches 0 (i.e., 0.0001 ), due to finite terms of the solution

$\% \% \% \% \%$

function $[$ pene] $=$ Pene_core_laminar(ratio_t2s, miu)

$\%$ This function returns sampling efficiency of a core sampling method

$\%$ Homogeneity of concentration distribution at the entrance and the laminar

$\%$ flow field should be guaranteed when applying this code 
$\%$ miu $=$ Pi*D*L/Q_tot

$\%$ ratio_t2s = transport flow rate / sample flow rate

$\% \mathrm{xi}=1 /($ ratio_t2s+1), where $\mathrm{xi}=$ core sample flow rate $/$ total flow rate

$x i=1 /($ ratio_t2s+1);

ri $=\operatorname{sqrt}(1-\operatorname{sqrt}(1-x i))$;

$d r=1 / 500 ; \% d r$ can be smaller if high resolution is needed

r_tuple = 0:dr:1;

$\% \mathrm{Ai}$ and lamda (dimensions 1x15)

$\%$ lamda is the solution for $\mathrm{M}(1 / 2-$ lamda/4, 1, lamda) $=0$

lamda $=[2.70436442,6.679031449,10.67337954,14.67107846,18.66987186,22.66914336$, 26.668662, 30.66832334, 34.66807382, 38.66788335, 42.66773381, 46.6676137, 50.6675154, 54.66743365, 58.66736475];

$\mathrm{Ai}=[1.47643540680257,-0.806123894775032,0.588762159116909,-0.475850417370689$, 0.405021794815585, -0.355756510893624, 0.319169069426987, -0.290735825223707, 0.267891171641597, -0.249062547548187, 0.233227814791694, -0.219691460536903, $0.207962410670599,-0.197683079668866,0.188586579953740]$;

$\%$ confluent hypergeometric function of the first kind

M_k=@(a,b,z,k) gamma(a+k)/gamma(a) * gamma(b)/gamma(b+k)* $z^{\wedge} k /$ factorial $(k)$;

$\mathrm{n}=\operatorname{zeros}\left(1\right.$, length $\left(r_{-}\right.$tuple $\left.)\right)$;

for $\mathrm{ii}=1$ :length(r_tuple) $\quad \%$ for each radius

$r=r_{-}$tuple(ii);

for $n n=1: 15 \%$ sufficient for convergence

$\mathrm{Mn}=0$;

$\%$ first $(\max (k)+1)$ terms of the expansion of the confluent hypergeometric function for $k=0: 20 \%$ sufficient for convergence

$M n=M n+M \_k\left(0.5-l a m d a(n n) / 4,1, \operatorname{lamda}(n n) * r^{\wedge} 2, k\right) ;$

end

En_r $=\exp \left(-\operatorname{lamda}(\mathrm{nn}) * \mathrm{r}^{\wedge} 2 / 2\right)$;

En_miu $=\exp \left(-\mathrm{miu}^{*} \operatorname{lamda}(\mathrm{nn})^{\wedge} 2 / 2\right)$;

$\% \mathrm{n}(\mathrm{miu}, \mathrm{r})$

$n(i i)=n(i i)+A i(n n) * M n * E n \_r * E n \_m i u$

end

end

$\%$ integration

if $r i<d r$

pene $=\mathrm{n}(1) ;$

else

$\mathrm{idx}=\mathrm{r}_{-}$tuple $<\mathrm{ri} ; \quad \%$ upper bound of the integration $r_{-}$a, in Eq. 6

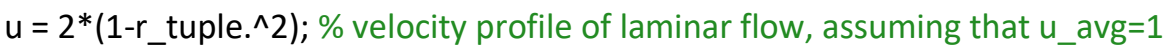


$\%$ average penetration at mu at the entrance of core sampling tube

pene $=$

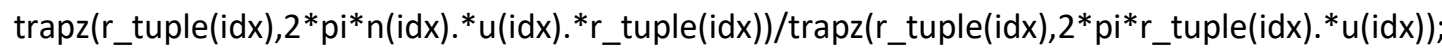

end

\section{References}

Alonso, M., Kousaka, Y., Hashimoto, T., Hashimoto, N. (1997). Penetration of Nanometer-Sized Aerosol Particles Through Wire Screen and Laminar Flow Tube. Aerosol Science and Technology, 27:471-480.

Bergman, T. L., Incropera, F. P., DeWitt, D. P., Lavine, A. S. (2011). Fundamentals of heat and mass transfer. John Wiley \& Sons.

Chen, R. Y. and Comparin, R. A. (1976). Deposition of aerosols in the entrance of a tube. Journal of Aerosol Science, 7:335-341.

Gormley, P. and Kennedy, M. (1948). Diffusion from a stream flowing through a cylindrical tube, Proceedings of the Royal Irish Academy. Section A: Mathematical and Physical Sciences, 53:163-169.

Kundu, P., Cohen, I., Dowling, D. (2012). Chapter 12-turbulence. Fluid Mechanics (Fifth Edition), Academic Press, Boston:541-620.

Mikhailov, M. D. and Cotta, R. M. (1997). Eigenvalues for the Graetz problem in slip-flow. International Communications in Heat and Mass Transfer, 24:449-451.

Sellars, J. R., Tribus, M., Klein, J. (1954). Heat transfer to laminar flow in a round tube or flat conduit: the Graetz probem extended. WADC Technical Report, 54.

Tan, C. W. and Hsu, C.-J. (1971). Diffusion of aerosols in laminar flow in a cylindrical tube. Journal of Aerosol Science, 2:117-124.

Wolfram, S. (1991). Mathematica: a system for doing mathematics by computer. Addison-Wesley. 